\title{
Xist at the edge: silencing at the nuclear periphery extends to $X$ inactivation
}

\author{
Justin Demmerle, Lothar Schermelleh \\ Micron Advanced Bioimaging Unit, Department of Biochemistry, University of Oxford, Oxford, OX1 3QU, UK \\ Correspondence to: Justin Demmerle; Lothar Schermelleh. Micron Advanced Bioimaging Unit, Department of Biochemistry, University of Oxford, \\ South Parks Road, Oxford, OX1 3QU, UK. Email: justin.demmerle@bioch.ox.ac.uk; lothar.schermelleh@bioch.ox.ac.uk.
}

Submitted Oct 18, 2016. Accepted for publication Oct 26, 2016.

doi: $10.21037 /$ tcr.2016.11.13

View this article at: http://dx.doi.org/10.21037/tcr.2016.11.13

The physical organization of chromatin in the nucleus plays a fundamental role in directing developmental cell fate decisions. The nuclear lamina in particular is a critical regulator of silencing specific genomic regions and establishing facultative heterochromatin (1). Chen et al. now demonstrate the connection between Xist-mediated silencing of the inactive $\mathrm{X}$ chromosome (XCI) and the repressive properties of the nuclear lamina (2). The absence of the inactive $\mathrm{X}$ chromosome has long been associated with tumors (3), and lately Xist, along with other long noncoding RNAs (lncRNAs), has been implicated in cancer etiology in addition to its role in dosage compensation $(4,5)$. These new findings emphasize the links between the Xist lncRNA, repressive epigenetic modifiers such as the polycomb repressive complexes (PRC1, PRC2), histone deacetylase 3 (HDAC3)-containing complexes, and the nuclear lamina, pointing towards new directions in the role of epigenetic silencing in translational cancer research.

The identity of the XCI as a lamina-associated domain is often visible to the eye, as labeled Barr bodies frequently occur at the nuclear periphery, and with reduced frequency at the nucleolus. However, a mechanistic description of this phenomenon has been lacking, with experiments focused on the biochemical establishment of repressive marks via PRC complexes in the absence of information about nuclear organization. Recent studies of the Xist proteome identified among other repressive epigenetic modifiers (PRC and SMRT complexes), architectural factors (SAF-A), RNAbinding proteins (SHARP and RBM15), and several nuclear lamina components including lamin B receptor (LBR) (6-8). LBR, an integral protein of the inner nuclear membrane, plays an essential role in developmentally regulated silencing through establishment and maintenance of facultative heterochromatin formation at the nuclear lamina by multiple epigenetic modifying complexes (9).

The authors analyzed Xist positioning and gene silencing in mouse ES cells treated with LBR siRNAs, and observed a concomitant loss of lamina-associated Xist signal and gene silencing activity. This behavior was also observed in LBR knockout ES cells. Using a cross-linking immunoprecipitation (CLIP) readout, they pinpointed LBR association to three main regions of the Xist transcript, including a SHARP-bound region previously identified as necessary for silencing. The use of the BoxB/ $\lambda \mathrm{N}$ tethering system to force association of Xist transcripts to the nuclear periphery provided clear evidence that the activity was reversible in the siLBR cells, as well as in mutants of the Xist-interacting LBR region and the LBR-binding region of Xist. The absence of Xist spreading to other regions of the inactive $\mathrm{X}$ chromosome was confirmed by RAP-DNA experiments showing decreased Xist RNA occupancy across the genomes of cells lacking the LBR-binding region of Xist $(\triangle \mathrm{LBS})$. However, knockdown of SHARP reduced silencing of the XCI despite maintenance of proximity to the lamina, indicating a multi-step mechanism, not simply lamina association, is necessary for silencing. Together, these findings led the authors to propose a model whereby Xist, silencing mechanisms, and the nuclear lamina are linked together for proper XCI silencing, but not independently sufficient for Xist spreading and silencing.

Several mechanistic questions are now raised by these studies. Super-resolution microscopy identifies on the order of 50-100 Xist foci per Barr body $(10,11)$ and many of these foci are not within a physical range (100s of nm) of the nuclear lamina to interact with LBR or other lamina-associated proteins (12). Thus, only a fraction of Xist molecules at any 


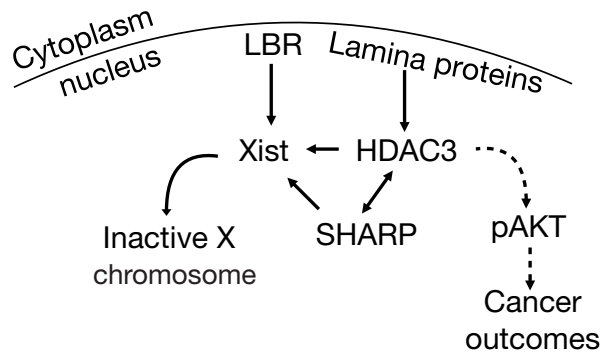

Figure 1 Interactions between Xist, the nuclear lamina, silencing factors, and cancer outcomes. Solid lines represent direct interactions, dashed lines represent indirect interactions. LBR, lamin B receptor.

given time will be mediating lamina-directed silencing. It is unknown whether each of these molecules will interact with the peripheral silencing compartment over the lifetime of an individual cell, or whether silencing activity, promoted by the lamina, will spread after nucleation at a subset of Xist foci. A finer time course of Xist association with the lamina, at superresolution level, may resolve this question. Additionally, a method of determining what proportion of Xist molecules interact biochemically with LBR in particular or the lamina in general, such as a DAM-ID/CLIP approach, could resolve the necessary questions about stoichiometry.

Another source of uncertainty is the timing and order of establishment of Xist interactions with the lamina. Repositioning experiments indicate that Xist- and LBRmediated silencing spread to loci such as Gpc4 over a 16-hour timespan following Xist induction (in the SHARP knockdown, and presumably in WT cells as well), and that knockdowns of LBR or the $\triangle \mathrm{LBS}$ and $\triangle \mathrm{A}$ mutants maintain a larger distance between the Gpc4 locus and Xist signal within this timeframe. The authors hypothesize that interactions between Xist and LBR are initiated by a dynamic sampling of the nuclear space by Xist-coated chromatin over a timespan of hours. However, lamina-associated domains are generally thought to be established across mitotic divisions, as the nuclear lamina re-forms in the proximity of regions containing sequence-specific factors that engage with lamina components, such as in the interaction between GAGA dinucleotide repeats, the GAGA-binding factor cKrox, the nuclear lamina protein Lap2B, and HDAC3 in the establishment of lamina-associated domains (13). Controlling for cell division, and extending the time-resolved analysis of Xist positioning in normal and mutant cells, perhaps with a live-cell approach using MS2-tagged Xist transcripts, would help answer this question.
The previous identification of a SHARP-HDAC3 complex as critical for Xist-mediated silencing (6) by the same group also reinforces the potential role of the nuclear lamina, as HDAC3 catalytic activity is enhanced at the nuclear lamina (14). Previous studies looking at the effect of various nuclear membrane components such as LMNB1, LBR, or emerin in developmental contexts make this discovery a logical progression of known lamina-mediated silencing phenomena. It would be informative to examine the role of nuclear lamina association in relation to other mechanisms, such as PRCmediated H3K27me3 deposition, RBM15-mediated $\mathrm{m}^{6} \mathrm{~A}$ methylation (15), and silencing at non-Xist regulated regions, to determine the degree to which lamina association is required for these events. For example, Xist could be tethered to another nuclear compartment, such as the nucleolus, via the BoxB/ $/ \mathrm{N}$ system, and the $\mathrm{XCI}$ assayed for silencing and PRC-mediated histone modifications to test the absolute necessity for lamina association in these processes. Further, assessing the effect of LBR depletion on the positioning behavior of both the active $\mathrm{X}$ chromosome and inducible Xist transgenes integrated on autosomes would help dissect the specificity of the proposed mechanism.

The work of Chen et al. is an important step towards establishing how the heterogeneous nuclear environment functions as a transcriptional regulator, as well as suggesting additional mechanisms of how Xist in particular, and lncRNAs in general, may establish developmentally regulated transcriptional silencing. As a similar process has recently been shown in C.elegans, this is likely an evolutionarily conserved mechanism coupling dosage compensation, developmentally regulated silencing, and the nuclear lamina (16). These findings have broad applicability to translational cancer research, particularly in breast cancer, where the Xist transcript shows increased transcriptional activity in cultured cells and patient samples (17). As the cooperative nature of HDAC3 complexes and Xist have recently been shown to regulate AKT phosphorylation in human breast cancer samples through a PHLPP1-dependent interaction (18), the connection to specific cancer-regulating mechanisms is clear. While this provides an informative network of interactions at the nuclear lamina involving Xist and HDAC3 leading to cancer-related outputs (Figure 1), many connections are indirect and merit further investigation. The ability to tether or release genomic regions via lncRNAs to the nuclear periphery or other compartments may become a useful tool in modulating gene expression across larger genomic regions, or provide a finetuning mechanism in genomic-based therapies. Further 
experiments, utilizing a suite of imaging and biochemical tools, will clarify this complex set of mechanisms and should point the way towards improved clinical outcomes through diagnostic and therapeutic strategies that leverage our growing knowledge of functional nuclear organization.

\section{Acknowledgements}

The authors thank Dr. Heather Coker for comments on the manuscript.

Funding: This work was supported by the Wellcome Trust Strategic Award 091911 to Micron Oxford and Lothar Schermelleh, and the National Institutes of Health OxfordCambridge Scholars Program to Justin Demmerle.

\section{Footnote}

Provenance: This is an invited Commentary commissioned by Section Editor Lichao Sun [State Key Laboratory of Molecular Oncology, National Cancer Center (NCC)/ Cancer Hospital, Chinese Academy of Medical Sciences (CAMS), Peking Union Medical College, Beijing, China]. Conflicts of Interest: The authors have no conflicts of interest to declare.

Comment on: Chen CK, Blanco M, Jackson C, et al. Xist recruits the $\mathrm{X}$ chromosome to the nuclear lamina to enable chromosome-wide silencing. Science 2016;354:468-72.

\section{References}

1. Bickmore WA. The spatial organization of the human genome. Annu Rev Genomics Hum Genet 2013;14:67-84.

2. Chen CK, Blanco M, Jackson C, et al. Xist recruits the $\mathrm{X}$ chromosome to the nuclear lamina to enable chromosomewide silencing. Science 2016;354:468-72.

3. Barr ML, Moore KL. Chromosomes, sex chromatin, and cancer. Proc Can Cancer Conf 1957;2:3-16.

4. Yildirim E, Kirby JE, Brown DE, et al. Xist RNA is a potent suppressor of hematologic cancer in mice. Cell 2013;152:727-42.

5. Seton-Rogers S. Non-coding RNAs: The cancer X factor. Nat Rev Cancer 2013;13:224-5.

6. McHugh CA, Chen CK, Chow A, et al. The Xist lncRNA interacts directly with SHARP to silence transcription through HDAC3. Nature 2015;521:232-6.

7. Chu C, Zhang QC, da Rocha ST, et al. Systematic discovery of Xist RNA binding proteins. Cell 2015;161:404-16.
8. Minajigi A, Froberg JE, Wei C, et al. A comprehensive Xist interactome reveals cohesin repulsion and an RNA-directed chromosome conformation. Science 2015;349. doi: 10.1126/ science.aab2276.

9. Solovei I, Wang AS, Thanisch K, et al. LBR and lamin $\mathrm{A} / \mathrm{C}$ sequentially tether peripheral heterochromatin and inversely regulate differentiation. Cell 2013;152:584-98.

10. Smeets D, Markaki Y, Schmid VJ, et al. Threedimensional super-resolution microscopy of the inactive $\mathrm{X}$ chromosome territory reveals a collapse of its active nuclear compartment harboring distinct Xist RNA foci. Epigenetics Chromatin 2014;7:8.

11. Sunwoo H, Wu JY, Lee JT. The Xist RNA-PRC2 complex at 20-nm resolution reveals a low Xist stoichiometry and suggests a hit-and-run mechanism in mouse cells. Proc Natl Acad Sci U S A 2015;112:E4216-25.

12. Cerase A, Smeets D, Tang YA, et al. Spatial separation of Xist RNA and polycomb proteins revealed by superresolution microscopy. Proc Natl Acad Sci U S A 2014;111:2235-40.

13. Zullo JM, Demarco IA, Piqué-Regi R, et al. DNA sequencedependent compartmentalization and silencing of chromatin at the nuclear lamina. Cell 2012;149:1474-87.

14. Demmerle J, Koch AJ, Holaska JM. The nuclear envelope protein emerin binds directly to histone deacetylase 3 (HDAC3) and activates HDAC3 activity. J Biol Chem 2012;287:22080-8.

15. Patil DP, Chen CK, Pickering BF, et al. m6A RNA methylation promotes XIST-mediated transcriptional repression. Nature 2016;537:369-373.

16. Snyder MJ, Lau AC, Brouhard EA, et al. Anchoring of Heterochromatin to the Nuclear Lamina Reinforces Dosage Compensation-Mediated Gene Repression. PLoS Genet 2016;12:e1006341.

17. Chaligné R, Popova T, Mendoza-Parra MA, et al. The inactive $\mathrm{X}$ chromosome is epigenetically unstable and transcriptionally labile in breast cancer. Genome Res 2015;25:488-503.

18. Huang YS, Chang CC, Lee SS, et al. Xist reduction in breast cancer upregulates AKT phosphorylation via HDAC3-mediated repression of PHLPP1 expression. Oncotarget 2016. [Epub ahead of print].

Cite this article as: Demmerle J, Schermelleh L. Xist at the edge: silencing at the nuclear periphery extends to $\mathrm{X}$ inactivation. Transl Cancer Res 2016;5(Suppl 6):S1189-S1191. doi: 10.21037/tcr.2016.11.13 